Invited paper

\title{
Dissipative solitons with a Lagrangian approach
}

\author{
Adrian Ankiewicz, Nail Akhmediev*, Natasha Devine \\ Optical Sciences Group, Research School of Physical Sciences and Engineering, The Australian National University, Canberra ACT 0200, Australia
}

Received 16 August 2006; revised 31 October 2006

Available online 12 January 2007

\begin{abstract}
We apply Lagrangian techniques to dissipative systems described by the complex Ginzburg-Landau equation (CGLE) that is used for modeling passively mode-locked fiber lasers and all-optical soliton transmission lines. In particular, using Lagrangian equations, we re-derive the known exact solutions of the CGLE. We also apply the technique to finding approximate solutions for pulsating solitons.
\end{abstract}

(c) 2006 Elsevier Inc. All rights reserved.

Keywords: Dissipative solitons; Fiber lasers; Lagrangian approach

\section{Introduction}

The dissipative soliton is a new concept that has been developed quite recently [1]. It includes ideas from three major sources, viz. standard soliton theory developed since the 1960s, principles from nonlinear dynamics theory and Prigogine's ideas of systems far from equilibrium. Physically speaking, the major part of standard soliton theory is the notion of the balance between dispersion and nonlinearity that allows stationary localized solutions to exist. However, for dissipative systems, we need to acknowledge that the major balance is between gain and loss which is necessary for the solitons to be stationary objects. Nonlinear dynamics inspires us with the idea of soliton bifurcations and the chaotic evolution of solitons. Finally, the theory of systems far from equilibrium tells us that solitons are self-organized formations requiring a continuous supply of energy. As soon as that supply finishes, a dissipative soliton ceases to exist. These ideas can be applied to various fiber optic devices such as passively mode-locked fiber lasers $[2,3]$ and high-density optical transmission lines [4]. A Lagrangian approach helps us in obtaining a deeper understanding of how these systems work and in solving particular problems related to fiber optic devices. This article provides a simple introduction to the Lagrangian approach applied to dissipative systems in general and to dissipative solitons in particular. The treat-

\footnotetext{
* Corresponding author.

E-mail address: nna124@rsphysse.anu.edu.au (N. Akhmediev).
}

ment is based on the cubic-quintic complex Ginzburg-Landau equation as it is a master equation for short pulse generation and propagation in nonlinear dissipative systems [2-4].

Generally defined, solitons are the simplest localized solutions of a certain class of partial differential evolution equations [5]. First, they were introduced as nonlinear 'modes' of the KdV equation, so that they allow us to solve initial value problems [6], when considered together with radiation waves. Indeed, when a system is integrable, its solutions can be constructed using the inverse scattering technique [5]. Further generalization to non-integrable systems [7] shows that localized traveling wave solutions can be found and analyzed using some basic approaches from nonlinear dynamics. One of the powerful techniques that can be applied to these systems is that which employs Hamiltonian equations. These are helpful in formulating the problem in term of a Hamiltonian [8] when the system is integrable, and in finding stationary solitons and stability regions for them if the problem is not integrable [9].

The notion of solitons can be generalized to cover dissipative systems [10]. Dissipative solitons are already well-defined objects in optics. Various techniques have been applied to investigate them, with numerical simulations being the most powerful. For dissipative systems, there are no conserved quantities that can be used to solve particular problems. In particular, Hamiltonian methods cannot be used here. However, for dissipative systems, we can write down the Lagrangian and use Lagrangian equations to derive soliton solutions. 
In this work, we apply Lagrangian principles to dissipative systems described by the complex cubic-quintic GinzburgLandau equation. We formulate Lagrangian equations for the CGLE and apply them to re-derive known solutions of this equation, and also analyze pulsating solutions that are only known from numerical simulations. In the latter case, solutions can be approximated by various localized trial functions that allow us to reduce the system to a finite-dimensional dynamical system. Solutions in the form of limit cycles for this dynamical system correspond to pulsating dissipative solitons. In the final section, we will present some new results for this topic.

The complex Ginzburg-Landau equation (CGLE) is a general model for dissipative systems, describing a vast variety of nonlinear phenomena in physics [11]. In particular, it describes pulse generation by passively mode-locked soliton lasers [12] and signal transmission in all-optical communication lines [13]. In dimensionless form, the CGLE is $[7,14]$

$$
\begin{aligned}
i \psi_{z} & +\frac{D}{2} \psi_{x x}+|\psi|^{2} \psi \\
& =-v|\psi|^{4} \psi+i \delta \psi+i \epsilon|\psi|^{2} \psi+i \beta \psi_{x x}+i \mu|\psi|^{4} \psi .
\end{aligned}
$$

Here the dispersion $D= \pm 1$, while $v$ represents the deviation from the Kerr-law response of the fiber. Nonlinear terms $\epsilon, \beta, \mu, \delta$ are defined in [14]. The application of the variational integral and Euler equations has been explained in [15, p. 276], where the use of the Lagrange density and its relation to the Euler-Lagrange equations for the Schroedinger equation and other conservative equations are also given (p. 314). We adopt the Lagrangian [action]

$L=\int_{-\infty}^{\infty} L_{d} \mathrm{~d} x$

where $L_{d}$ is the Lagrangian density. Here, we have

$$
\begin{aligned}
L= & -\frac{i}{2} \int_{-\infty}^{\infty}\left(\psi^{*} \frac{\partial \psi}{\partial z}-\psi \frac{\partial \psi^{*}}{\partial z}\right) \mathrm{d} x \\
& +\frac{D}{2} P-\frac{1}{2} S_{4}-\frac{v}{3} S_{6}
\end{aligned}
$$

for field $\psi(x, z)$.

Here, we have defined

$S_{n}=\int_{-\infty}^{\infty}|\psi|^{n} \mathrm{~d} x$ and $P=\int_{-\infty}^{\infty}\left|\frac{\partial \psi}{\partial x}\right|^{2} \mathrm{~d} x$.

Now, $Q=S_{2}$ is the total pulse energy. The rate of change of $Q$ is [14]

$Q_{z}=\frac{\mathrm{d} Q}{\mathrm{~d} z}=2\left(\delta Q-\beta P+\epsilon S_{4}+\mu S_{6}\right)$.

Now, if we apply the Euler-Lagrange equations to the CGLE, we obtain

$\frac{\partial}{\partial z} \frac{\partial L_{d}}{\partial \psi_{z}^{*}}+\frac{\partial}{\partial x} \frac{\partial L_{d}}{\partial \psi_{x}^{*}}-\frac{\partial L_{d}}{\partial \psi^{*}}=R$, where a subscript $x$ or $z$ means derivative w.r.t. that variable, * indicates complex conjugate and $R$ indicates the dissipative terms of the CGLE, viz.,

$R=i\left[\delta \psi+\epsilon|\psi|^{2} \psi+\mu|\psi|^{4} \psi+\beta \psi_{x x}\right]$

For a stationary solution (i.e., not a pulsating solution [24], for example), the energy $Q$ is constant and so the term $Q_{z}=$ $\mathrm{d} Q / \mathrm{d} z$ is also zero (see p. 271 of [14]). This condition constrains the solutions.

Of course, if we have a stationary state, then the soliton profile does not depend on $z$. The solution written as a complex function in general form

$\psi(x, z)=B(x) \exp (-i \theta(z))$

has the complex amplitude function $B(x)$ that does not depend on $z$. Thus $|\psi|=|B(x)|$. The phase function $\theta(z)=\omega z$ is a linear function of $z$, so $\theta^{\prime}(z)=\omega$. If we use this ansatz, then the first term in the Lagrangian reduces to

$-\omega \int_{-\infty}^{\infty}|B(x)|^{2} \mathrm{~d} x=-\omega Q$.

For the (real) term $P$, we have

$P=\int_{-\infty}^{\infty}\left|B^{\prime}(x)\right|^{2} \mathrm{~d} x=-\int_{-\infty}^{\infty} B^{*}(x) B^{\prime \prime}(x) \mathrm{d} x$,

assuming a localized function, i.e., that $B(x)$ vanishes at $\pm \infty$. The signs of the parameters in Eq. (1) are defined by the physics of the optical system (see [14]). In optics, we usually have $\delta<0, \mu<0, \epsilon>0, \beta>0$, so then the 'gain' term is $\epsilon S_{4}$. In some cases, we can regard the stationary solution as the one which takes minimum energy from the environment. However, in general it is not sufficient to have a solution constrained by $\mathrm{d} Q / \mathrm{d} z=0$ while retaining one free parameter, and then maximizing the 'gain' relative to that parameter.

Up till now, the Lagrangian has mostly been used to find approximate solutions of conservative systems, like the nonlinear Schroedinger equation (NLSE) or the NLSE with a non-Kerrlaw term (i.e., $v \neq 0$ ) (see [16-18]). For a trial solution containing parameters $f_{j}, j=1,2, \ldots$, the standard variational approach can be modified to allow for dissipative terms like self-steepening and magnetic effects (see [19-21]). We have, from Eq. (3),

$\frac{\mathrm{d}}{\mathrm{d} z}\left(\frac{\partial L}{\partial f_{z}}\right)-\frac{\partial L}{\partial f}=2 \operatorname{Re}\left(\int_{-\infty}^{\infty} R \frac{\partial \psi^{*}}{\partial f} \mathrm{~d} x\right)$

for each parameter $f$. Our purpose here in this part is quite different in that we seek exact solutions of the CGLE, which is a dissipative system involving a balance between gain and loss in various parts of a pulse. Of course, the pulse could be a signal traveling along an optical fiber, an electric or magnetic field distribution, or function giving a distribution of temperature, pressure or number of particles [1].

If we have an ansatz, then the derivative of the Lagrangian w.r.t. any parameter should be equal to an integral involving the 
dissipative terms. This may or may not be zero. We now show how this works. It is thus a new way to find solutions of the CGLE.

\section{General formalism for exact solutions}

In this section, we apply the Lagrangian formalism to find stationary soliton solutions, i.e., solutions which have the form of Eq. (7), of the CGLE. Then, using Eq. (10), we have, for a general parameter $f$ :

$$
\begin{aligned}
& \frac{\partial L}{\partial f}-\frac{\mathrm{d}}{\mathrm{d} z}\left(\frac{\partial L}{\partial f_{z}}\right)=2 \operatorname{Re} \int_{-\infty}^{\infty}\left(\frac{1}{i \psi^{*}} \frac{\partial \psi^{*}}{\partial f}\right) \\
& \quad \times\left[\delta|B|^{2}+\epsilon|B|^{4}+\mu|B|^{6}+\beta B_{x x} B^{*}\right] \mathrm{d} x .
\end{aligned}
$$

Now, suppose, the parameter $f$ in Eq. (11) is $f=\theta(z)$. Noting that

$$
\frac{1}{i \psi^{*}} \frac{\partial \psi^{*}}{\partial \theta}=1
$$

from Eq. (11), we get

$$
\begin{aligned}
\frac{\mathrm{d} Q}{\mathrm{~d} z} & =2 \operatorname{Re} \int_{-\infty}^{\infty}\left[\delta|B|^{2}+\epsilon|B|^{4}+\mu|B|^{6}+\beta B_{x x} B^{*}\right] \mathrm{d} x \\
& =2\left(\delta Q-\beta P+\epsilon S_{4}+\mu S_{6}\right) .
\end{aligned}
$$

This result is nothing other than the so-called "balance equation" [14] for energy $Q$. Hence, using the phase function as a parameter in the Lagrangian equation verifies Eq. (4). Plainly, the r.h.s. is zero for a stationary solution. The energy of the stationary solution is constant, but this does not indicate the conservation of energy which applies for the NLSE. We recall that, for the NLSE, each conserved quantity is related to a symmetry of the equation (Noether's theorem), and that energy conservation is related to a phase shift symmetry (p. 29 of [14]). In a somewhat analogous manner, for the CGLE here, it is the phase term parameter that results in the energy being constant for the stationary solution.

In contrast to conservative cases, stationary solutions in dissipative systems can have a phase chirp. Thus, the function $B$ in general is complex rather than real. A wide range of exact solutions of the CGLE can be expressed using the ansatz

$B(x)=a(x) \exp [i d \log [a(x)]]$,

where the amplitude function $a(x)$ is real and even. This ansatz explicitly uses the parameter $d$ to characterize the phase chirp. We assume that the parameters $f_{j}$, other than $d$, are contained in $a(x)$ implicitly. Now if $f$ is a parameter other than $d$, then

$\frac{1}{i \psi^{*}} \frac{\partial \psi^{*}}{\partial f}=-\frac{i+d}{a} \frac{\partial a}{\partial f}$

and

$$
\begin{aligned}
\frac{\partial L}{\partial f}= & 4 d \int_{0}^{\infty} \frac{\partial a}{\partial f}\left[\frac{\beta}{a(x)}\left(1+d^{2}\right)\left[a^{\prime}(x)\right]^{2}\right. \\
& \left.-\delta a(x)-\epsilon a^{3}(x)-\mu a^{5}(x)\right] \mathrm{d} x .
\end{aligned}
$$

The Lagrangian is given by Eq. (3) with the use of (7), where $P$ can now be simplified to

$P=2\left(1+d^{2}\right) \int_{0}^{\infty}\left[a^{\prime}(x)\right]^{2} \mathrm{~d} x$.

The parameter $d$ needs to be considered separately. We have

$\frac{1}{i \psi^{*}} \frac{\partial \psi^{*}}{\partial d}=-\log [a(x)]$

and

$$
\begin{aligned}
\frac{\partial L}{\partial d}= & -4 \int_{0}^{\infty}\left(\delta a^{2}(x)+\epsilon a^{4}(x)+\mu a^{6}(x)\right) \log [a(x)] \mathrm{d} x \\
& +4 \beta \int_{0}^{\infty}\left[a^{\prime}(x)\right]^{2}\left(1+\left(1+d^{2}\right) \log [a(x)]\right) \mathrm{d} x .
\end{aligned}
$$

Using Eq. (14) and further specifying $a(x)=b \operatorname{sech}(c x)$, we find the Lagrangian:

$L=\frac{b^{2}}{45 c}\left[-30 b^{2}-16 b^{4} v+15\left(c^{2}\left(1+d^{2}\right) D-6 \omega\right)\right]$.

The balance equation is now given by

$Q_{z}=\frac{1}{15 c}\left[4 b^{2}\left(-5 \beta c^{2}\left(1+d^{2}\right)+15 \delta+10 b^{2} \epsilon+8 b^{4} \mu\right)\right]$.

Derivatives of the Lagrangian with respect to parameters involved can further be calculated. We derive, using Eqs. (4) and (16),

$\frac{\partial L}{\partial b}=\frac{d}{b} Q_{z}=0$,

since the energy, $Q$, does not change for a stationary solution. Hence

$5\left(c^{2}\left(1+d^{2}\right) D-6 \omega\right)-20 b^{2}-16 b^{4} v=0$,

and then

$\frac{\partial L}{\partial d}=\frac{2}{3} b^{2} c d D$.

However,

$\frac{1}{i \psi^{*}} \frac{\partial \psi^{*}}{\partial c}=(d+i) x \tanh (c x)$

which gives

$\frac{\partial L}{\partial c}=\frac{2 d b^{2}}{c^{2}}\left[\delta+\epsilon \frac{b^{2}}{3}+\mu \frac{8 b^{4}}{45}-\frac{2}{3} \beta c^{2}\left(1+d^{2}\right)\right]$.

Further, we need the derivative of the Lagrangian with respect to the parameter $d$. It follows, from Eq. (18),

$\frac{1}{i \psi^{*}} \frac{\partial \psi^{*}}{\partial d}=-\log (b \operatorname{sech}(c x))$

which, using Eq. (19), gives

$\frac{\partial L}{\partial d}=-\left[\delta d_{2}+\epsilon d_{4}+\mu d_{6}+\beta d_{s}\right]$ 
where the coefficients $d_{i}$ are given by

$$
\begin{aligned}
d_{2} & =4 \int_{0}^{\infty} a^{2} \log (b \operatorname{sech}(c x)) \mathrm{d} x \\
& =\frac{4 b^{2}}{c}(-1+\log (2 b)), \\
d_{4} & =4 \int_{0}^{\infty} a^{4} \log (b \operatorname{sech}(c x)) \mathrm{d} x \\
& =\frac{4 b^{4}}{9 c}(-5+6 \log (2 b)), \\
d_{6} & =4 \int_{0}^{\infty} a^{6} \log (b \operatorname{sech}(c x)) \mathrm{d} x \\
& =\frac{8 b^{6}}{225 c}(-47+60 \log (2 b)),
\end{aligned}
$$

and, finally, for $d_{s}$, we have

$$
\begin{aligned}
d_{s} & =-4 \int_{0}^{\infty}\left[a^{\prime}(x)\right]^{2}\left(1+\left(1+d^{2}\right) \log (b \operatorname{sech}(c x))\right) \mathrm{d} x \\
& =\frac{4}{9} b^{2} c\left(1+4 d^{2}-3\left(1+d^{2}\right) \log (2 b)\right) .
\end{aligned}
$$

Now, we are equipped with all necessary relations in order to apply the general formalism to find various particular solutions of the CGLE. The solutions are limited by our ansatz (Eqs. (7) and (14)), of course. For the cubic CGLE, it gives the complete set of bright solutions of the CGLE, while, for the cubic-quintic CGLE, it allows us to pick up only a small subclass of the solutions. However, thus far, this ansatz provides the only known way to obtain exact solutions for the cubicquintic CGLE. What follows below are a few examples which are particular cases of this general ansatz.

\section{Basic NLSE soliton}

For illustrative purposes we consider the case when all coefficients in (1) are zero except $D=1$. The nonlinear Schrödinger equation is a particular case of the CGLE (1) when the dissipative terms and the term with $v$ are zero. This is the simplest case where we can apply the Lagrangian approach. Using the ansatz with $v=0, d=0$, i.e.,

$\psi=b \operatorname{sech}(c x) \exp (-i \theta(z))$,

with arbitrary parameters $b$ and $c$, we find that the Lagrangian is

$L=\frac{b^{2}}{3 c}\left(-2 b^{2}+c^{2}-6 \theta^{\prime}(z)\right)$.

So, the condition

$\frac{\partial L}{\partial b}=0$

leads to

$-4 b^{2}+c^{2}-6 \theta^{\prime}(z)=0$, while

$$
\frac{\partial L}{\partial c}=0 \Rightarrow 2 b^{2}+c^{2}+6 \theta^{\prime}(z)=0 .
$$

Now $Q_{z}=0$ is automatically satisfied, so we have only one degree of freedom. We get $b=c$ and $\theta^{\prime}(z)=\omega=-c^{2} / 2$. This is the exact one-soliton solution of the NLSE, $\psi=$ $c \operatorname{sech}(c x) \exp \left(i c^{2} z / 2\right)$.

\section{Cubic CGLE chirp-free soliton}

Here $d=0, D=1$ and $\epsilon=2 \beta, v=\mu=0$. Using the same ansatz as above, $L$ is the same as above. Now $Q_{z}=0$ requires $4 b^{2} \beta-\beta c^{2}+3 \delta=0$, so we no longer have a degree of freedom in the solution:

$\theta^{\prime}(z)=\omega=\frac{\delta}{2 \beta}, \quad b=c=\sqrt{-\delta / \beta}$.

These are the correct values, as seen from Eq. (13.31) on p. 279 of [14], which was derived in quite a different way.

\section{Solution with arbitrary amplitude}

We know [23] (see also Chapter 13 of [14]) that when $\delta=0$, both cubic and cubic-quintic CGLE admit soliton solutions with arbitrary amplitude. Thus, setting $\delta=0, \nu=\mu=0$, $D=1$, and using relation (13.24) of [14] to relate $\beta$ and $\epsilon$, we can directly solve the equations involving $Q_{z}$ and the derivatives w.r.t. $b$ and $c$ to get

$d=\frac{\sqrt{1+4 \beta^{2}}-1}{2 \beta}$,

$\omega=-d c^{2} \frac{1+4 \beta^{2}}{2 \beta}$

and

$b^{2}=\frac{d c^{2}}{2 \epsilon} \sqrt{1+4 \beta^{2}}$,

with $c$ being arbitrary. Setting $b=G F$ and $c=G$ reproduces the known results (Section 13.4.2 of [14]). Substitution shows that these values also satisfy the ' $d$ ' equation.

\section{General cubic CGLE soliton}

Here we only set $v=\mu=0$. We use Eq. (11) for

$\frac{\partial L}{\partial b}, \quad \frac{\partial L}{\partial c} \quad$ and $\quad Q_{z}$

to get

$b=\sqrt{\frac{3 \delta(D+\beta d)}{\beta-2 D \epsilon-3 \beta d \epsilon}}$,

$c=\sqrt{\frac{3 \delta(\epsilon d-1)}{\left(1+d^{2}\right)(2 D \epsilon+\beta(3 \epsilon d-1))}}$

and

$\theta^{\prime}(z)=\frac{\delta(4 \beta d+D(3+d \epsilon))}{4 D \epsilon+2 \beta(3 d \epsilon-1)}$. 
On converting, the $D=1$ set agrees with the values given in Section 13.4.1 of [14]. We then use

$$
\frac{\partial L}{\partial d}=-2 b^{2} c d / 3
$$

to get the equation for $d$ :

$$
(2 \beta-D \epsilon) d^{2}+3 d(D+2 \beta \epsilon)+2(D \epsilon-2 \beta)=0 .
$$

Solving this quadratic equation for $D=1$ gives the correct values for $d$ (i.e., Eq. (13.17) of [14]). This shows that, to obtain $d=0$ (chirpless solution), we need $D \epsilon=2 \beta$. This explains why we cannot just have arbitrary values of $\epsilon, \beta$ for the solution of Eq. (38), which assumes $d=0$.

\section{Rational solution}

Here we set $D=1, \delta=0$ and seek a rational solution which has the form of Eq. (14) with

$|\psi|^{2}=f(x)=a^{2}(x)=\frac{f_{1}}{1+k f_{1}^{2} x^{2}}$.

Then

$\frac{1}{i \psi^{*}} \frac{\partial \psi^{*}}{\partial f_{1}}=\frac{a^{2}}{2 f_{1}^{2}}(d+i)\left(k f_{1}^{2} x^{2}-1\right)$,

while

$\frac{1}{i \psi^{*}} \frac{\partial \psi^{*}}{\partial k}=\frac{a^{2}}{2}(d+i) f_{1} x^{2}$

and

$\frac{1}{i \psi^{*}} \frac{\partial \psi^{*}}{\partial d}=-\frac{1}{2} \log (f(x))$.

Hence

$$
\begin{aligned}
\frac{\partial L}{\partial k}= & f_{1} \operatorname{Re} \int_{-\infty}^{\infty} a^{2}(i+d) x^{2} \\
& \times\left[\delta|\psi|^{2}+\epsilon|\psi|^{4}+\mu|\psi|^{6}+\beta \psi_{x x} \psi^{*}\right] \mathrm{d} x,
\end{aligned}
$$

and similarly for the other 2 parameters. We find $\partial L / \partial f_{1}=$ $-(\pi d / 4 \sqrt{k})\left(\epsilon+f_{1} \mu\right)$, while $\partial L / \partial d$ involves $\log$ terms. Clearly, $|\psi|^{2 n}=f(x)^{n}, n=1,2, \ldots$.

With this solution, we have $\delta=0$ and hence the frequency offset $\omega$ is zero. Also, $\mu$ is not arbitrary, but is related to $v$ by a particular expression (Eq. (13.20) of [14]). The Lagrangian is

$L=\frac{\pi f_{1}}{16 \sqrt{k}}\left(-4+f_{1}\left(k+d^{2} k-2 v\right)\right)$,

so that

$\frac{\partial L}{\partial f_{1}}=\frac{\pi}{8 \sqrt{k}}\left(-2+f_{1}\left(k+d^{2} k-2 v\right)\right)$,

etc. Solving the equations for $k$ and $f_{1}$ simultaneously gives

$k=\frac{2 v}{3+8 \beta d-d^{2}}, \quad f_{1}=\frac{\left(3+8 \beta d-d^{2}\right)(d \epsilon-1)}{2 v(1+\beta d)\left(1+d^{2}\right)}$.

When we additionally use the equation giving $Q_{z}$, we get the quadratic equation for $d$ and arrive at the correct value (Eq. (13.17) of [14]). On conversion, $k, f_{1}$ also agree with published values.

\section{Quintic CGLE chirp-free soliton}

One of the soliton solutions of the cubic-quintic CGLE is the chirp-free $(d=0)$ soliton. It appears when we take $\epsilon=2 \beta$ and $\mu=2 \beta v$ [14]. For convenience, we take $\beta>0, v>0, \delta<0$. Writing $\exp (-i \omega z)$ for the $z$-dependence, we use the ansatz

$a(x)=\sqrt{c}[1+\sqrt{g} \cosh (x \sqrt{2 c})]^{-1 / 2}$

for the amplitude function. Then we try to find the correct values of $c, g, \omega$ for the solution. The result for $S_{2}$ is

$S_{2}=Q=2 \sqrt{\frac{2 c}{g-1}} A$,

where $A=\arctan (\sqrt{1-2 /(1+\sqrt{g})})$.

We also need the expression for $S_{4}$

$S_{4}=\sqrt{2}\left(\frac{c}{g-1}\right)^{3 / 2}[\sqrt{g-1}-2 A]$

and

$S_{6}=\frac{1}{\sqrt{2}}\left(\frac{c}{g-1}\right)^{5 / 2}[-3 \sqrt{g-1}+2(2+g) A]$.

The remaining term is

$P=-\frac{1}{2 \sqrt{2}}\left(\frac{c}{g-1}\right)^{3 / 2}[\sqrt{g-1}-2 g A]$.

Then, setting the algebraic part of $Q_{z}$ to zero gives

$3 g-3-4 c v=0$,

so $g=1+\frac{4}{3} c v$. Then the transcendental part (with function arctan) gives

$\beta c+2 \delta=0 \Rightarrow c=-2 \frac{\delta}{\beta}(>0)$.

Now taking $D=1$ and using $\partial L / \partial c=0$ shows that

$\omega=\frac{\delta}{2 \beta}$.

With these values

$\frac{\partial L}{\partial c}=0, \quad \frac{\partial L}{\partial g}=0$,

so we have an extremum of $L$. Thus $g=1-8 \delta v /(3 \beta)(>1)$.

A similar example, with a different derivation, can be seen from Eqs. (13.57) (on p. 287) and (13.18) $(d=0)$ of [14].

\section{Lagrangian technique and pulsating dissipative solitons}

Thus far, we have applied a Lagrangian approach to derive exact solutions of various forms of the CGLE. However, not all soliton solution admit an exact analytical form. Some of the solutions of the cubic-quintic GLE are known only numerically [24]. These include stationary solitons as well as pulsating ones. The Lagrangian approach can also be used to find the best approximation for these objects when we do not expect to find exact solutions. Approximating stationary solitons with trial functions is a trivial task. This can be done in a similar 
fashion to examples of conservative systems [25]. In this section, we will apply the technique to finding approximate CGLE soliton solutions which are not necessarily stationary. It is well known [24] that the CGLE admits pulsating solitons, in addition to solutions in the form of stationary solutions. These correspond to limit cycles of the dissipative dynamical system rather than to fixed points. Thus, these are more complicated objects in the infinite-dimensional phase space. There are no known exact solutions for these formations. However, pulsating solutions can be approximated using various localized $z$-dependent functions with several parameters and the so-called "method of moments." Thus, in contrast to the previous sections, we are concerned here with approximate solutions rather than exact ones. In particular, in this section, we show how the Lagrangian method can be used to derive the results of the "method of moments' [22]. To be specific, we will use a Gaussian-type ansatz

$$
\begin{aligned}
\psi(x, z)= & A \sqrt{\frac{Q(z)}{f(z)}} \exp \left(-\frac{x^{2}}{f^{2}(z)}-\frac{x^{4}}{m f^{4}(z)}\right. \\
& \left.+i c(z) x^{2}-i \theta(z)\right),
\end{aligned}
$$

where

$A^{2}=2 /\left(e^{m / 4} \sqrt{m} K_{1 / 4}\left(\frac{m}{4}\right)\right)$,

with $K$ being the modified Bessel function of the second kind. The soliton profile is localized in $x$ and has explicit $z$ dependence. The pulse evolution is described through the variation of total energy $Q(z)$, pulse width, $f(z)$, chirp factor $c(z)$ and the overall phase, $\theta(z)$.

For clarity, we restrict ourselves to the lowest-order Gaussian and thus take $m=\infty$, so $A=(2 / \pi)^{1 / 4}$. This leads to the Lagrangian $L$, where

$$
\begin{aligned}
\frac{L}{Q}= & \frac{D}{2 f^{2}}+D \frac{c^{2} f^{2}}{2}-\frac{Q}{2 \sqrt{\pi} f} \\
& -\frac{2 \nu Q^{2}}{3 \sqrt{3} \pi f^{2}}+\frac{1}{4} f^{2} c^{\prime}(z)-\theta^{\prime}(z) .
\end{aligned}
$$

Then, for any function depending on $\theta$ in the exponential as in Eq. (65), we have

$\frac{\partial L}{\partial \theta}=0$.

However,

$\frac{\partial L}{\partial \theta^{\prime}(z)}=-Q(z)$

so that

$\frac{\mathrm{d}}{\mathrm{d} z}\left(\frac{\partial L}{\partial \theta^{\prime}(z)}\right)=-Q^{\prime}(z)$

Thus, we reproduce balance equation (4) using the explicit dependence on phase. Consequently, Eq. (69) is the same as the derivative of energy w.r.t. $z$ from the method of moments [22].
Now $\partial L / \partial c=D c f^{2} Q$. This quantity was denoted by $I_{3} / i$ in [22]. Further $\partial L / \partial c^{\prime}(z)=\frac{1}{4} f^{2} Q$ (which was denoted by $\left.I_{2}\right)$. Also

$\frac{1}{i \psi^{*}}\left(\frac{\partial \psi^{*}}{\partial c}\right)=-x^{2}$,

so that

$$
\begin{aligned}
& \frac{\mathrm{d}}{\mathrm{d} z}\left(\frac{1}{4} f^{2} Q\right)-c f^{2} Q \\
& \quad=2 \operatorname{Re} \int_{-\infty}^{\infty}\left(x^{2}\right)\left[\delta B^{2}+\epsilon B^{4}+\mu B^{6}+\beta \psi_{x x} \psi^{*}\right] \mathrm{d} x
\end{aligned}
$$

where $B=|\psi|$. Here, the left hand side equals $(\mathrm{d} / \mathrm{d} z) I_{2}+i I_{3}$ so we have reproduced the second-order moment equation from [22]. Hence

$$
\begin{aligned}
f^{\prime}(z)= & \frac{2 \beta}{f(z)}+2 D c f-2 \beta c^{2}(z) f^{3} \\
& -\frac{\epsilon Q}{2 \sqrt{\pi}}-\frac{4 \mu Q^{2}}{3 \sqrt{3} \pi f}
\end{aligned}
$$

(see Eq. (A10) in [22]). Now

$\frac{1}{i \psi^{*}}\left(\frac{\partial \psi^{*}}{\partial Q}\right)=-\frac{i}{2 Q}$,

thus leading to

$\frac{\partial L}{\partial Q}=0$

while

$\frac{1}{i \psi^{*}}\left(\frac{\partial \psi^{*}}{\partial f}\right)=\frac{i}{2 f^{3}}\left(f^{2}-4 x^{2}\right)$

so $\partial L / \partial f \neq 0$. Using these relations, we find

$$
\begin{aligned}
c^{\prime}(z)= & \frac{2 D}{f(z)^{4}}-2 D c^{2}-8 \beta \frac{c}{f^{2}(z)} \\
& -\frac{Q}{\sqrt{\pi} f^{3}(z)}-\frac{8 v Q^{2}}{3 \sqrt{3} \pi f^{4}(z)}
\end{aligned}
$$

(see Eq. (A13) in [22]) and

$\theta^{\prime}(z)=\frac{D}{f^{2}(z)}-2 \beta c-\frac{5 Q}{4 \sqrt{\pi} f(z)}-\frac{8 v Q^{2}}{3 \sqrt{3} \pi f^{2}(z)}$

Equations (72), (76) and (77) comprise the three-dimensional dynamical system that governs the evolution of the soliton parameters. This dynamical system is the same as that obtained using the method of moments [22], and this is also true when we use $m=1$. It has solutions in the form of fixed points and limit cycles. The former correspond to stationary solitons, the latter to pulsating solitons. To increase the accuracy of the approximation, we can use a trial function with more parameters and derive equations for higher-order moments. If the chirp term is changed to $c(z) x^{n}$, then

$$
\frac{1}{i \psi^{*}}\left(\frac{\partial \psi^{*}}{\partial c}\right)=-x^{n} \text {. }
$$


Then we have $\partial L / \partial c \sim c f^{2 n-2} Q$ and $\partial L / \partial c^{\prime}(z) \sim f^{n} Q$, and hence an $n$ th-order moment-type equation is obtained. So the reduced dynamical system for solitons that was obtained using the method of moments can also be obtained using the Lagrangian approach. When using the same trial function, we obtain the same dynamical system. In this sense, the two techniques are equivalent although the principles used to obtain them are different.

\section{Conclusions}

In conclusion, we have shown that the technique of minimization of the Lagrangian can be used for the dissipative systems described by the complex cubic-quintic Ginzburg-Landau equation. The technique allows us to derive exact solutions of this equation as well as approximate solutions in the form of pulsating solitons. In the former case, we recover the complete set of known solutions of the CGLE while in the latter case we show that the results obtained using the Lagrangian approach are the same as those obtained using the "method of moments."

\section{Acknowledgment}

The authors gratefully acknowledge the support of the Australian Research Council.

\section{References}

[1] N. Akhmediev, A. Ankiewicz, Dissipative solitons in the complex Ginzburg-Landau and Swift-Hohenberg equations, in: N. Akhmediev, A. Ankiewicz (Eds.), Dissipative Solitons, Springer, Heidelberg, 2005.

[2] N. Akhmediev, J.M. Soto-Crespo, M. Grapinet, Ph. Grelu, Dissipative soliton interactions inside a fiber laser cavity, Opt. Fiber Technol. 11 (2005) 209.

[3] J.N. Kutz, Mode-locking of fiber lasers via nonlinear mode coupling, in: N. Akhmediev, A. Ankiewicz (Eds.), Dissipative Solitons, Springer, Heidelberg, 2005.

[4] U. Peschel, D. Michaelis, Z. Bakonyi, G. Onishchukov, F. Lederer, Dynamics of dissipative temporal solitons, in: N. Akhmediev, A. Ankiewicz (Eds.), Dissipative Solitons, Springer, Heidelberg, 2005.

[5] M.J. Ablowitz, P.A. Clarkson, Solitons, Nonlinear Evolution Equations and Inverse Scattering, London Mathematical Society Lecture Notes Series, vol. 149, Cambridge Univ. Press, Cambridge, 1991.
[6] N.J. Zabusky, M.D. Kruskal, Interaction of 'solitons' in a collisionless plasma and the recurrence of initial states, Phys. Rev. Lett. 15 (1965) 240243

[7] G.P. Agrawal, Nonlinear Fiber Optics, second ed., Academic Press, San Diego, CA, 1995.

[8] L.D. Faddeev, L.A. Takhtajan, Hamiltonian Methods in the Theory of Solitons, Springer, Berlin, 1987.

[9] N. Akhmediev, A. Ankiewicz, R. Grimshaw, Hamiltonian versus energy diagrams in soliton theory, Phys. Rev. E 59 (5) (1999) 6088-6096.

[10] N. Akhmediev, A. Ankiewicz (Eds.), Dissipative Solitons, Springer, Heidelberg, 2005.

[11] I.S. Aronson, L. Kramer, The world of the complex Ginzburg-Landau equation, Rev. Mod. Phys. 74 (2002) 99.

[12] E.P. Ippen, Principles of passive mode locking, Appl. Phys. B 58 (1994) 159.

[13] Z. Bakonyi, et al., Dissipative solitons and their critical slowing down near a supercritical bifurcation, J. Opt. Soc. Am. B 19 (2002) 487.

[14] N. Akhmediev, A. Ankiewicz, Solitons: Nonlinear Pulses and Beams, Chapman \& Hall, London, 1997.

[15] P.M. Morse, H. Feshbach, Methods of Theoretical Physics, McGraw-Hill, 1953.

[16] A.D. Bonderson, M. Lisak, D. Anderson, Soliton perturbations: A variational principle for the soliton parameters, Phys. Scripta 20 (1979) 479.

[17] A. Ankiewicz, N. Akhmediev, G.D. Peng, P.L. Chu, Limitations of the variational approach for soliton propagation in nonlinear couplers, Opt. Comm. 103 (1993) 410-416.

[18] G.D. Peng, P.L. Chu, A. Ankiewicz, Soliton propagation in saturable nonlinear fibre couplers, Int. J. Nonlinear Opt. Phys. 3 (1994) 69-87.

[19] A.D. Boardman, L. Velasco, Gyroelectric cubic-quintic dissipative solitons, IEEE J. Select. Top. Quantum Electron. 12 (2006) 388.

[20] M. Manousakis, et al., Propagation of chirped solitary pulses in optical transmission lines: Perturbed variational approach, Opt. Comm. 213 (2002) 293-299.

[21] S. Chavez Cerda, et al., A variational approach of nonlinear dissipative pulse propagation, Eur. J. Phys. D 1 (1998) 313-316.

[22] E. Tsoy, A. Ankiewicz, N. Akhmediev, Dynamical models for dissipative localized waves of the complex Ginzburg-Landau equation, Phys. Rev. E 73 (2006) 036621.

[23] N.N. Akhmediev, V.V. Afanasjev, Novel arbitrary-amplitude soliton solutions of the cubic-quintic complex Ginzburg-Landau equation, Phys. Rev. Lett. 75 (12) (1995) 2320-2323.

[24] N. Akhmediev, J.M. Soto-Crespo, G. Town, Pulsating solitons, chaotic solitons, period doubling, and pulse coexistence in mode-locked lasers: Complex Ginzburg-Landau equation, Phys. Rev. E 63 (2001) 056602.

[25] D. Anderson, Variational approach to nonlinear pulse propagation in optical fibers, Phys. Rev. A 27 (6) (1983) 3135-3145. 